PROCEEDINGS OF THE

AMERICAN MATHEMATICAL SOCIETY

Volume 138, Number 9, September 2010, Pages 3229-3239

S 0002-9939(10)10338-4

Article electronically published on April 9, 2010

\title{
LIOUVILLIAN FIRST INTEGRALS FOR LIÉNARD POLYNOMIAL DIFFERENTIAL SYSTEMS
}

\author{
J. LLIBRE AND C. VALLS \\ (Communicated by Yingfei Yi)
}

\begin{abstract}
We characterize the Liouvillian first integrals for the Liénard polynomial differential systems of the form $x^{\prime}=y, y^{\prime}=-c x-f(x) y$, with $c \in \mathbb{R}$ and $f(x)$ is an arbitrary polynomial. For obtaining this result we need to find all the Darboux polynomials and the exponential factors of these systems.
\end{abstract}

\section{InTRODUCTION AND STATEMENT OF THE MAIN RESULTS}

One of the more classical problems in the qualitative theory of planar differential systems depending on parameters is to characterize the existence or not of first integrals.

We consider the system

$$
x^{\prime}=y, \quad y^{\prime}=-c x-f(x) y,
$$

which we call the generalized classical Liénard differential system, where $x$ and $y$ are complex variables and the prime denotes derivative with respect to the time $t$, which can be either real or complex. Such differential systems appear in several branches of the sciences, such as biology, chemistry, mechanics, electronics, etc. For $c=1$ the Liénard differential systems (1) are called the classical Liénard systems.

The main objective of this paper is to study the Liouvillian first integrals of systems (11) depending on the polynomial function $f(x)$ and on $c \in \mathbb{R}$.

Let $U \subset \mathbb{C}^{2}$ be an open set. We say that the nonconstant function $H: \mathbb{C}^{2} \rightarrow \mathbb{C}$ is a first integral of the polynomial vector field $X$ on $U$ if $H(x(t), y(t))=$ constant for all values of $t$ for which the solution $(x(t), y(t))$ of $X$ is defined on $U$. Clearly $H$ is a first integral of $X$ on $U$ if and only if $X H=0$ on $U$.

A Liouvillian first integral is a first integral $H$ which is a Liouvillian function, that is, roughly speaking, which can be obtained "by quadratures" of elementary functions. For a precise definition, see 13. The study of the Liouvillian first integrals is a classical problem of the integrability theory of the differential equations which goes back to Liouville; see for details again [13.

As far as we know, the Liouvillian first integral of some multi-parameter family of planar polynomial differential systems has only been classified for the LotkaVolterra system; see [1, 5, 8, 9, 10, 11.

Received by the editors September 10, 2009 and, in revised form, December 16, 2009.

2010 Mathematics Subject Classification. Primary 37K10, 34D30.

Key words and phrases. Darboux polynomials, exponential factors, Liouvillian first integrals, Liénard polynomial differential systems. 
Our main result is the classification of all Liouvillian integrable systems (10).

Theorem 1. The unique Liouvillian first integrals $H=H(x, y)$ of the Liénard polynomial differential system (11) are:

(a) $H=c x^{2}+y^{2}$ if $f(x)=0$;

(b) $H=y+\int f(x) d x$ if $c=0$;

(c) $H=\left(\frac{1}{2}(1-\sqrt{1+4 \alpha}) x+y\right)^{-1+\sqrt{1+4 \alpha}}\left(\frac{1}{2}(1+\sqrt{1+4 \alpha}) x+y\right)^{1+\sqrt{1+4 \alpha}}$ with $\alpha=-c / f(0)^{2}$ if $f(x)=f(0) \neq 0$; and

(d) $H=e^{f^{\prime}(0)^{2} x^{2}+2 f^{\prime}(0) y}\left(c+f^{\prime}(0) y\right)^{-2 c}$ if $f(x)=f^{\prime}(0) x \neq 0$.

For proving Theorem 1 we need to characterize the Darboux polynomial and the exponential factors of system (1). The scheme of the proof of Theorem 1 is given in Section 2 ,

\section{The SCheme of the PRoof of Theorem 1}

System (11) with $f(x)=0$ becomes $x^{\prime}=y, y^{\prime}=-c x$, which has the first integral $H=c x^{2}+y^{2}$.

System (11) with $c=0$ becomes, after the change of time $d s=y d t$, the system $\dot{x}=1, \dot{y}=-f(x)$, where now the dot denotes derivative with respect to $s$. This system has the first integral $H=y+F(x)$, where $F(x)=\int_{0}^{x} f(s) d s$. We will write

$$
f(x)=\sum_{j=0}^{n} a_{j} x^{j} \quad \text { and hence } \quad F(x)=\sum_{j=0}^{n} \frac{a_{j}}{j+1} x^{j+1} .
$$

In view of this we can always assume that $f(x) \not \equiv 0$ and that $c \neq 0$. Furthermore it was proved in [7] (see Lemma 3) that a Liénard differential system (11) with $c \neq 0$ after a rescaling of the variables $(x, y, t)$ becomes another Liénard differential system (10) with $c= \pm 1$.

Furthermore if $f(x)=f(0) \neq 0$, then system (11) is linear, that is,

$$
x^{\prime}=y, \quad y^{\prime}=-c x-f(0) y .
$$

We write $c=-\alpha f(0)^{2}, \alpha \in \mathbb{R} \backslash\{0\}$. Then equation (3) becomes

$$
x^{\prime}=y, \quad y^{\prime}=\alpha f(0)^{2} x-f(0) y .
$$

Doing the rescaling $(X, Y, T)=(f(0) x, y, f(0) t)$, system (4) becomes

$$
x^{\prime}=y, \quad y^{\prime}=\alpha x-y,
$$

where we have written again $(x, y, t)$ instead of $(X, Y, T)$. It is easy to check that system (5) has the first integral given in statement (c) of Theorem 1.

Let $n$ be the degree of $f(x)$. If $n=1$, then $f(x)=f(0)+f^{\prime}(0) x$. Additionally, if $f(0)=0$, then system (1) becomes $x^{\prime}=y, y^{\prime}=-c x-f^{\prime}(0) x y$. Again it is easy to check that this system has the first integral given in statement (d) of Theorem 1,

In short, from now on, we can always assume the following assumptions: (6)

$c= \pm 1 ; \quad f(x)$ has degree at least one; if $f(x)$ has degree one, then $f(0) \neq 0$.

A first integral which is a polynomial in the variables $x$ and $y$ is called a polynomial first integral of system (11).

Proposition 2. System (1) under assumptions (2) and (6) has no polynomial first integrals. 
The polynomial first integrals of system (11) when instead of $c x$ there is a polynomial $g(x)$ and having a unique remarkable value are studied in [4].

Let $h(x, y) \in \mathbb{C}[x, y] \backslash \mathbb{C}$. As usual $\mathbb{C}[x, y]$ denotes the ring of all complex polynomials in the variables $x$ and $y$. We say that $h=0$ is an invariant algebraic curve of the vector field $X$ associated to system (1) if it satisfies

$$
y \frac{\partial h}{\partial x}-(c x+f(x) y) \frac{\partial h}{\partial y}=K h,
$$

the polynomial $K=K(x, y) \in \mathbb{C}[x, y]$ is called the cofactor of $h=0$ and has degree at most $n$. We also say that $h$ is a Darboux polynomial of system (11). Note that a polynomial first integral is a Darboux polynomial with zero cofactor.

The invariant algebraic curves are important becase a sufficient number of them forces the existence of a first integral. This result is the basis of the Darboux theory of integrability; see for instance [3, 6.

Proposition 3. System (11) under assumptions (2) and (6) has no Darboux polynomials.

Propositions 2 and 3 are immediate corollaries of the following theorem of Odani (see [12] for its proof).

Theorem 4. If a polynomial Liénard system

$$
x^{\prime}=y, \quad y^{\prime}=-f(x) y-g(x)
$$

satisfies $f, g \neq 0, \operatorname{deg} f \geq \operatorname{deg} g$, and $g / f \neq$ constant, then it has no algebraic solution curves.

An exponential factor $E$ of system (1) is a function of the form $E=\exp (g / h) \notin \mathbb{C}$ with $g, h \in \mathbb{C}[x, y]$ satisfying

$$
y \frac{\partial E}{\partial x}-(c x+f(x) y) \frac{\partial E}{\partial y}=L E,
$$

for some polynomial $L=L(x, y)$ of degree at most $n$, called the cofactor of $E$.

The existence of exponential factors $\exp (\mathrm{g} / \mathrm{h})$ is due to the fact that the multiplicity of the invariant algebraic curve $h=0$ is larger than 1 ; for more details see [2.

Theorem 5. System (11) under assumptions (2) and (6) has the exponential factors $e^{x^{j}}$ for $j=1, \ldots, n$, and $e^{\left(y x^{k-1}+\int f(x) x^{k-1} d x\right) /(k-1)}$ with cofactors $x^{j-1} y$ for $j=$ $1, \ldots, n$, and $y^{2} x^{k-2}-c x^{k} /(k-1)$ for $k=2, \ldots, n$, respectively.

The last result presented in this section is:

Theorem 6. System (11) under assumptions (2) and (6) has no Liouvillian first integrals.

Clearly from the results presented in this section Theorem 1 follows.

The rest of the paper is as follows. In Section 3 we give auxiliary results that will be used throughout this paper. In Section 4 we prove Theorem 5, and in Section 5 we prove Theorem 6 . 


\section{Preliminary Results}

By definition a complex planar polynomial differential system or simply a polynomial system will be a differential system of the form

$$
x^{\prime}=P(x, y), \quad y^{\prime}=Q(x, y),
$$

where the dependent variables $x$ and $y$ are complex and the independent variable (the time) $t$ is real or complex, and $P$ and $Q$ are polynomials in the variables $x$ and $y$ with complex coefficients. Throughout this paper $m=\max \{\operatorname{deg} P, \operatorname{deg} Q\}$ will denote the degree of the polynomial system. The vector field $X$ associated to system (7) is defined by

$$
X=P \frac{\partial}{\partial x}+Q \frac{\partial}{\partial y}
$$

We say that $h=0$ is an invariant algebraic curve of the vector field $X$ if it satisfies

$$
X h=P \frac{\partial h}{\partial x}+Q \frac{\partial h}{\partial y}=K h ;
$$

where the polynomial $K=K(x, y) \in \mathbb{C}[x, y]$ is called the cofactor of $h=0$ and has degree at most $m-1$.

Proposition 7. The following statements hold:

(a) If $E=\exp (g / h)$ is an exponential factor for the polynomial system (7) and $h$ is not a constant polynomial, then $h=0$ is an invariant algebraic curve.

(b) Eventually $e^{x^{k}}$ for $k=1,2, \ldots$ can be exponential factors, coming from the multiplicity of the infinite invariant straight line.

For a geometrical meaning of the exponential factors and a proof of Proposition 7 see [2].

The next result summarizes the main results about the Darboux theory of integrability that we shall use in this paper.

Theorem 8. Suppose that the polynomial vector field $X$ of degree $m$ defined in $\mathbb{C}^{2}$ admits $p$ invariant algebraic curves $f_{i}=0$ with cofactors $K_{i}$, for $i=1, \ldots, p$ and $q$ exponential factors $E_{j}=\exp \left(g_{j} / h_{j}\right)$ with cofactors $L_{j}$, for $j=1, \ldots, q$. Then the following statements hold:

(a) There exist $\lambda_{i}, \mu_{j} \in \mathbb{C}$ not all zero such that

$$
\sum_{i=1}^{p} \lambda_{i} K_{i}+\sum_{j=1}^{q} \mu_{j} L_{j}=0
$$

if and only if the function of Darboux type

$$
f_{1}^{\lambda_{1}} \cdots f_{p}^{\lambda_{p}} E_{1}^{\mu_{1}} \cdots E_{q}^{\mu_{q}}
$$

is a first integral of the vector field $X$.

(b) There exist $\lambda_{i}, \mu_{j} \in \mathbb{C}$ not all zero such that

$$
\sum_{i=1}^{p} \lambda_{i} K_{i}+\sum_{j=1}^{q} \mu_{j} L_{j}=-\operatorname{div}(P, Q)
$$

if and only if the function of Darboux type (9) is an integrating factor of the vector field $X$. 
(c) If $p+q=[m(m+1) / 2]+1$, then there exist $\lambda_{i}, \mu_{j} \in \mathbb{C}$ not all zero such that (8) holds. If $p+q=m(m+1) / 2$, then there exist $\lambda_{i}, \mu_{j} \in \mathbb{C}$ not all zero such that either (8) or (10) holds.

A nonconstant complex function $R: \mathbb{C}^{2} \rightarrow \mathbb{C}$ is an integrating factor of the polynomial vector field $X$ on $U$ if one of the following three equivalent conditions holds:

$$
\frac{\partial(R P)}{\partial x}=-\frac{\partial(R Q)}{\partial y}, \quad \operatorname{div}(R P, R Q)=0, \quad X R=-R \operatorname{div}(P, Q),
$$

on $U$. As usual the divergence of the vector field $X$ is given by

$$
\operatorname{div}(P, Q)=\frac{\partial P}{\partial x}+\frac{\partial Q}{\partial y}
$$

To prove the results related with Liouvillian first integrals we use the following result proved in [13].

Theorem 9. The polynomial differential system (7) has a Liouvillian first integral if and only if it has an integrating factor of Darboux type.

\section{Proof of Theorem 5}

Let $E=\exp (g / h)$ be an exponential factor of system (11). In view of Propositions 7,3 and 2 we have that $h$ can be taken as a constant and $E=\exp (g)$. Let

$$
L=L(x, y)=\sum_{k=0}^{n} \sum_{j=0}^{k} \beta_{j, k-j} x^{j} y^{k-j}, \quad \beta_{j, k-j} \in \mathbb{C}
$$

be the cofactor associated to $E$. Then if we define

$$
G=E e^{\beta_{1,0}(y+F(x)) / c-\sum_{k=1}^{n} \beta_{k-1,1} x^{k} / k}=E e^{T},
$$

we claim that $G=e^{H}$ is an exponential factor of system (1) with cofactor

$$
L_{1}=L_{1}(x, y)=\beta_{0,0}+\sum_{k=2}^{n} \sum_{j=0, j \neq k-1}^{k} \beta_{j, k-j} x^{j} y^{k-j}, \quad \beta_{j, k-j} \in \mathbb{C} .
$$

Now we prove the claim. We have that

$$
\begin{aligned}
& y \frac{\partial G}{\partial x}-(c x+f(x) y) \frac{\partial G}{\partial y} \\
& =y \frac{\partial E}{\partial x} e^{T}+y \frac{\partial T}{\partial x} E e^{T}-(c x+f(x) y) \frac{\partial E}{\partial y} e^{T}-(c x+f(x) y) \frac{\partial T}{\partial y} E e^{T} \\
& =\left(L-y \sum_{k=1}^{n} \beta_{k-1,1} x^{k-1}-\beta_{1,0} x\right) E e^{T}=L_{1} G .
\end{aligned}
$$

This proves the claim.

Since $G=e^{H}$ is an exponential factor of system (11) with cofactor $L_{1}$ we obtain that

$$
y \frac{\partial H}{\partial x}-(c x+f(x) y) \frac{\partial H}{\partial y}=L_{1}(x, y) .
$$


Evaluating (12) at $x=y=0$ we get that $\beta_{0,0}=0$ and $H$ satisfies

$$
y \frac{\partial H}{\partial x}-(c x+f(x) y) \frac{\partial H}{\partial y}=\sum_{k=2}^{n} \sum_{j=0, j \neq k-1}^{k} \beta_{j, k-j} x^{j} y^{k-j}, \quad \beta_{j, k-j} \in \mathbb{C} .
$$

If $n=1$, then from (13) we get that $H$ must be a polynomial first integral, in contradiction with Proposition 2. Therefore we can assume that $n \geq 2$. We write $H$ as a polynomial in the variable $y$ as $H=\sum_{j=0}^{r} H_{j}(x) y^{j}$. Without loss of generality we can assume that $H_{r}(x) \neq 0$. We rewrite (13) as

$$
\begin{aligned}
& \sum_{j=0}^{r} H_{j}^{\prime}(x) y^{j+1}-c x \sum_{j=1}^{r} j H_{j}(x) y^{j-1}-f(x) \sum_{j=0}^{r} j H_{j}(x) y^{j} \\
& =\sum_{l=2}^{n} \sum_{m=0, m \neq 1}^{l} \beta_{l-m, m} x^{l-m} y^{m}=\sum_{l=2}^{n} \beta_{l, 0} x^{l}+\sum_{m=2}^{n} y^{m} \sum_{l=m}^{n} \beta_{l-m, m} x^{l-m} .
\end{aligned}
$$

Now we consider two cases.

Case 1: $r \geq n$. In this case, computing in (14) the coefficient of $y^{r+1}$ we get that $H_{r}^{\prime}(x)=0$, that is, $H_{r}(x)=\gamma_{r} \in \mathbb{C} \backslash\{0\}$. Now we will show that if we write

$$
H=\gamma_{r} y^{r}+\sum_{j=0}^{r-1} H_{r-h}(x) y^{r-j}
$$

then

$$
H_{r-j}(x)=\gamma_{r} \frac{a_{n}^{j}}{j !(n+1)^{j}} x^{j(n+1)} \prod_{i=0}^{j-1}(r-i)+\text { l.o.t., } \quad \text { for } j=1, \ldots, r-1,
$$

where l.o.t. means lower order terms in $x$.

For $j=1$, computing the coefficient of $y^{r}$ in (14) (note that since $f(x)$ has degree $n$ in $x$, the right-hand side of equation (14) contains only lower order terms in $x$ ), we get that

$$
H_{r-1}^{\prime}(x)-f(x) r H_{r}(x)=0, \quad \text { that is, } \quad H_{r-1}^{\prime}(x)=\gamma_{r} r f(x) .
$$

Integrating it we obtain

$$
H_{r-1}(x)=\gamma_{r} r F(x)+\text { constant }=\gamma_{r} \frac{a_{n}}{n+1} r x^{n+1}+\text { l.o.t. },
$$

which coincides with (15) for $j=1$. Now we assume that (15) is true for $j=0, \ldots, L$ with $L<r-1$ and we will prove it for $j=L+1$. Computing the terms in (14) (note again that since $f(x)$ has degree $n$ in $x$, the right-hand side of equation (14) contain only lower order terms in $x$ ) with $y^{r-L}$ we get

$$
H_{r-L-1}^{\prime}(x)=c x(r-L+1) H_{r-L+1}(x)+f(x)(r-L) H_{r-L}(x) .
$$

Now using the induction hypothesis and since $x^{(L-1)(n+1)+1}$ belongs to the lower terms in comparison with $x^{L(n+1)}$ we obtain that

$$
\begin{aligned}
H_{r-L-1}^{\prime}(x) & =f(x)(r-L) \frac{\gamma_{r} a_{n}^{L}}{(n+1)^{L} L !} x^{L(n+1)} \prod_{i=0}^{L-1}(r-i)+\text { l.o.t. } \\
& =\frac{\gamma_{r} a_{n}^{L+1}}{(n+1)^{L} L !} x^{L(n+1)+n} \prod_{i=0}^{L}(r-i)+\text { l.o.t. }
\end{aligned}
$$


Now integrating the previous equation we obtain

$$
\begin{aligned}
H_{r-L-1}(x) & =\frac{\gamma_{r} a_{n}^{L+1}}{(n+1)^{L} L !(L+1)(n+1)} x^{(L+1)(n+1)} \prod_{i=0}^{L}(r-i)+\text { l.o.t. } \\
& =\frac{\gamma_{r} a_{n}^{L+1}}{(n+1)^{L+1}(L+1) !} x^{(L+1)(n+1)} \prod_{i=0}^{L}(r-i)+\text { l.o.t. },
\end{aligned}
$$

which is equation (15) with $j=L+1$. This completes the proof of (15).

From (15) with $j=r-1$ we obtain

$$
H_{1}(x)=\frac{\gamma_{r} a_{n}^{r-1}}{(r-1) !(n+1)^{r-1}} x^{(r-1)(n+1)} \prod_{i=0}^{r-2}(r-i)+\text { l.o.t. }
$$

Then computing the coefficient of $y^{0}$ in (14) we get

$$
-c x H_{1}(x)=\sum_{l=2}^{n} \beta_{l, 0} x^{l} .
$$

Since $n \geq 2$ and $r>n-1 \geq 1$ we have that $(r-1)(n+1)+1>n$. Computing the coefficient of $x^{(r-1)(n+1)+1}$ in the previous equality, we get

$$
\frac{c \gamma_{r} a_{n}^{r-1}}{(r-1) !(n+1)^{r-1}} \prod_{i=0}^{r-2}(r-i)=0
$$

which is a contradiction because the right-hand side of this expression is not zero.

Case 2: $r \leq n-1$. We first assume that $r \geq 2$ and we will reach a contradiction. We claim that (14) becomes

$$
\begin{aligned}
& \sum_{j=0}^{r} H_{j}^{\prime}(x) y^{j+1}-c x \sum_{j=1}^{r} j H_{j}(x) y^{j-1}-f(x) \sum_{j=0}^{r} j H_{j}(x) y^{j} \\
& =\sum_{l=2}^{n} \beta_{l, 0} x^{l}+\sum_{m=2}^{r+1} y^{m} \sum_{l=m}^{n} \beta_{l-m, m} x^{l-m} .
\end{aligned}
$$

Indeed, since all the coefficients with $y^{m}$ for $m=r+2, \ldots, n$ in (14) only appear in the right-hand side, we have that

$$
\sum_{m=r+2}^{n} y^{m} \sum_{l=m}^{n} \beta_{l-m, m} x^{l-m}=0 .
$$

This implies that (16) holds. Computing the coefficient of $y^{r+1}$ in (16) we get that

$$
\begin{aligned}
& H_{r}^{\prime}(x)=\sum_{l=r+1}^{n} \beta_{l-r-1, r+1} x^{l-r-1}, \quad \text { that is, } \\
& H_{r}(x)=c_{r}+\sum_{l=r+1}^{n} \frac{\beta_{l-r-1, r+1}}{l-r} x^{l-r}=\sum_{l=r}^{n} \tilde{\beta}_{l} x^{l-r},
\end{aligned}
$$

where $\tilde{\beta}_{r}=c_{r} \in \mathbb{C}$ and $\tilde{\beta}_{l}=\beta_{l-r-1, r+1} /(l-r)$ for $l=r+1, \ldots, n$. Without loss of generality and since $H_{r}(x) \neq 0$ we denote by $l^{*}$ the greatest integer of $\{r, \ldots, n\}$ such that $\tilde{\beta}_{l^{*}} \neq 0$. Then it is clear that

$$
H_{r}(x)=\tilde{\beta}_{l^{*}} l^{l^{*}-r}+\text { l.o.t. }
$$


We claim that

$$
H_{r-j}(x)=\frac{\tilde{\beta}_{l^{*}} a_{n}^{j}}{\prod_{i=1}^{j}\left(i(n+1)+l^{*}-r\right)} x^{j(n+1)+l^{*}-r} \prod_{i=0}^{j-1}(r-i)+\text { l.o.t. },
$$

for $j=1, \ldots, r-1$.

Computing the coefficient of $y^{r}$ in (16) we get

$$
H_{r-1}^{\prime}(x)-f(x) r H_{r}(x)=\sum_{l=r}^{n} \beta_{l-r, r} x^{l-r} .
$$

Since $l^{*} \geq r \geq 2$, the terms $x^{n-r}$ belong to the lower terms in comparison with $x^{n-r+l^{*}}$. Then we obtain that

$$
H_{r-1}^{\prime}(x)=a_{n} r \tilde{\beta}_{l^{*}} x^{n+l^{*}-r}+\text { l.o.t. }
$$

Integrating this last expression we get

$$
H_{r-1}(x)=\frac{\tilde{\beta}_{l^{*}} a_{n}}{n+1+l^{*}-r} x^{n+1+l^{*}-r} r+\text { l.o.t. }
$$

which coincides with (17) with $j=1$.

Now we assume that (17) is true for $j=1, \ldots, L$ with $1 \leq L<r-1$ and we will prove it for $j=L+1$. Computing the coefficient of $y^{r-L}$ in (16) we get

$H_{r-L-1}^{\prime}(x)-c x(r-L+1) H_{r-L+1}(x)-f(x)(r-L) H_{r-L}(x)=\sum_{l=r-L}^{n} \beta_{l-r+L, r-L} x^{l-r+L}$.

Now using the induction hypothesis and since $x^{(L-1)(n+1)+1+l^{*}-r}$ and $x^{n-r+L}$ belong to the lower terms in comparison with $x^{L(n+1)+l^{*}-r}$ (note that $L \geq 1$ ), we obtain that

$$
\begin{aligned}
H_{r-L-1}^{\prime}(x) & =a_{n} x^{n}(r-L) \frac{\tilde{\beta}_{l^{*}} a_{n}^{L}}{\prod_{i=1}^{L}\left(i(n+1)+l^{*}-r\right)} x^{L(n+1)+l^{*}-r} \prod_{i=0}^{L-1}(r-i)+\text { l.o.t. } \\
& =\frac{\tilde{\beta}_{l^{*}} a_{n}^{L+1}}{\prod_{i=1}^{L}\left(\left(i(n+1)+l^{*}-r\right)\right.} x^{L(n+1)+l^{*}-r+n} \prod_{i=0}^{L}(r-i)+\text { l.o.t. }
\end{aligned}
$$

Now integrating the previous equation we obtain

$$
\begin{aligned}
H_{r-L-1}(x) & =\frac{\tilde{\beta}_{l^{*}} a_{n}^{L+1} x^{(L+1)(n+1)+l^{*}-r}}{\left((L+1)(n+1)+l^{*}-r\right) \prod_{i=1}^{L}\left(i(n+1)+l^{*}-r\right)} \prod_{i=0}^{L}(r-i)+\text { l.o.t. } \\
& =\frac{\tilde{\beta}_{l^{*}} a_{n}^{L+1}}{\prod_{i=1}^{L+1}\left(i(n+1)+l^{*}-r\right)} x^{(L+1)(n+1)+l^{*}-r} \prod_{i=0}^{L}(r-i)+\text { l.o.t. }
\end{aligned}
$$

which is equation (17) with $j=L+1$. This proves the claim done in (17).

From (17) with $j=r-1$ we obtain

$$
H_{1}(x)=\frac{\tilde{\beta}_{l^{*}} a_{n}^{r-1}}{\prod_{i=1}^{r-1}\left(i(n+1)+l^{*}-r\right)} x^{(r-1)(n+1)+l^{*}-r} \prod_{i=0}^{r-2}(r-i)+\text { l.o.t. }
$$

Then we have that the coefficient of $y^{0}$ in (16) satisfies

$$
-c x H_{1}(x)=\sum_{l=2}^{n} \beta_{l, 0} x^{l} .
$$


Since $r \geq 2$ we have that $(r-1)(n+1)+l^{*}-r+1=(r-1) n+l^{*} \geq n+l^{*} \geq n+r \geq n+2$ and the coefficient of $x^{(r-1) n+l^{*}}$ in the previous equation becomes

$$
\frac{c \tilde{\beta}_{l^{*}} a_{n}^{r-1}}{\prod_{i=1}^{r-1}\left(i(n+1)+l^{*}-r\right)} \prod_{i=0}^{r-2}(r-i)=0,
$$

a contradiction because the right-hand side is not zero.

This shows that $r<2$. So we have two cases.

Case 1: $r=0$. In this case, $H(x, y)=H_{0}(x)$, and it follows from (16) that

$$
y H_{0}^{\prime}(x)=\sum_{l=2}^{n} \beta_{l, 0} x^{l}
$$

which yields that $H_{0}(x)$ is a constant. Hence, from (11), we obtain that $E=e^{-T}$ with

$$
T=\frac{\beta_{1,0}}{c}(y+F(x))-\sum_{k=1}^{n} \frac{\beta_{k-1,1}}{k} x^{k} .
$$

Consequently we get that $e^{y+F(X)}=e^{\left(y x^{k-1}+\int f(x) x^{k-1} d x\right) /(k-1)}$ for $k=2$, and $e^{x^{j}}$ for $j=1, \ldots, n$ are exponential factors. It is easy to check that their cofactors are $c x$ and $x^{j-1} y$, respectively.

Case 2: $r=1$. Then $H(x, y)=H_{0}(x)+y H_{1}(x)$ and from (12) and (16) becomes

(18) $y H_{0}^{\prime}(x)+y^{2} H_{1}^{\prime}(x)-(c x+f(x) y) H_{1}(x)=\sum_{l=2}^{n} \beta_{l, 0} x^{l}+y^{2} \sum_{l=2}^{n} \beta_{l-2,2} x^{l-2}=L_{1}$.

Computing the coefficient of $y^{2}$ in (18) we get

$$
H_{1}^{\prime}(x)=\sum_{l=2}^{n} \beta_{l-2,2} x^{l-2}, \quad \text { i.e. } \quad H_{1}(x)=\beta^{*}+\sum_{l=2}^{n} \frac{\beta_{l-2,2}}{l-1} x^{l-1} \quad \text { with } \quad \beta^{*} \in \mathbb{C} .
$$

Furthermore the coefficient of $y$ in (18) gives

$$
H_{0}^{\prime}(x)=f(x) H_{1}(x)=f(x)\left(\beta^{*} \sum_{l=2}^{n} \frac{\beta_{l-2,2}}{l-1} x^{l-1}\right) .
$$

Finally the coefficient of $y^{0}$ in (18) gives

$$
-c\left(\beta^{*} x+\sum_{l=2}^{n} \frac{\beta_{l-2,2}}{l-1} x^{l}\right)=\sum_{l=2}^{n} \beta_{l, 0} x^{l} .
$$

Consequently

$$
\beta^{*}=0 \quad \text { and } \quad \beta_{l, 0}=-\frac{c \beta_{l-2,2}}{l-1}, \quad l=2, \ldots, n .
$$

In short we have

$$
H(x, y)=\sum_{l=2}^{n} \frac{\beta_{l-2,2}}{l-1}\left(\int f(x) x^{l-1} d x+y x^{l-1}\right)
$$

and

$$
L_{1}(x)=\sum_{l=2}^{n} \beta_{l-2,2}\left(-\frac{c}{l-1} x^{l}+y^{2} x^{l-2}\right) .
$$

Hence the proof of Theorem 5 follows easily. 


\section{Proof of Theorem 6}

Assume that we have system (11) under the assumptions (2) and (6). Then by Proposition 3 this system has no Darboux polynomials, and by Theorem 5 it has $2 n-2$ exponential factors $E_{i}$ for $i=1, \ldots, 2 n-2$. We denote the cofactors of the exponential factors by $L_{i}$.

In order that system (1) has a Liouvillian first integral, by Theorem 9, system (11) must have an integrating factor of Darboux type (see (9)). From Theorem 8(b), system (11) has an integrating factor of Darboux type if and only if

$$
\sum_{i=1}^{2 n-2} \mu_{i} L_{i}=f(x), \quad \mu_{i} \in \mathbb{C} .
$$

Hence by using Theorem 5 the equality in (19) becomes

$$
y \sum_{j=1}^{n} \mu_{j} x^{j-1}+\sum_{k=2}^{n} \mu_{n+k}\left(y^{2} x^{k-2}-\frac{c}{k-1} x^{k}\right)=f(x) .
$$

This equality is not possible because the left-hand side is independent of $y$. This ends the proof of Theorem 6 .

\section{ACKNOWLEDGEMEnTs}

The first author has been supported by the grants MCYT/FEDER MTM200803437 and CIRIT 2009SGR 410. The second author has been partially supported by FCT through CAMGDS, Lisbon.

\section{REFERENCES}

[1] L. Cairó, H. Giacomini and J. Llibre, Liouvillian first integrals for the planar Lotka-Volterra system, Rend. Circ. Mat. Palermo 52 (2003), 389-418. MR2029552 (2004j:34094)

[2] C. Christopher, J. Llibre and J.V. Pereira, Multiplicity of invariant algebraic curves and Darboux integrability, Pacific J. Math. 229 (2007), 63-117. MR2276503(2008f:34065)

[3] G. Darboux, Mémoire sur les équations différentielles algébriques du premier ordre et du premier degré (Mélanges), Bull. Sci. Math. 2ème. série 2 (1878), 60-96; 123-144; 151-200.

[4] B. Garcia, J. Llibre and J.S. Pérez del Río, On the polynomial differential systems having polynomial first integrals, preprint, 2009.

[5] S. Labrunie, On the polynomial first integrals of the $(a, b, c)$ Lotka-Volterra system, J. Math. Phys. 37 (1996), 5539-5550. MR1417159 (98a:34012)

[6] J. Llibre, Integrability of polynomial differential systems, Handbook of Differential Equations, Ordinary Differential Equations, Eds. A. Canada, P. Drabek and A. Fonda, Elsevier (2004), pp. 437-533. MR.2166493 (2006e:34077)

[7] J. Llibre and C. Valls, On the local analytic integrability at the singular point of a class of Liénard analytic differential sytems, Proc. Amer. Math. Soc. 138 (2010), 253-261.

[8] J. Moulin Ollagnier, Polynomial first integrals of the Lotka-Volterra system, Bull. Sci. Math. 121 (1997), 463-476. MR1477795 (99h:34012)

[9] J. Moulin Ollagnier, Rational integration of the Lotka-Volterra system, Bull. Sci. Math. 123 (1999), 437-466. MR1712673 (2001d:34017)

[10] J. Moulin Ollagnier, Liouvillian integration of the Lotka-Volterra system, Qual. Theory Dyn. Syst. 2 (2001), 307-358. MR1913289 (2003f:34014)

[11] J. Moulin Ollagnier, Corrections and complements to: "Liouvillian integration of the LotkaVolterra system" [Qual. Theory Dyn. Syst. 2 (2001), 307-358], Qual. Theory Dyn. Syst. 5 (2004), 275-284. MR2275441 
[12] K. Odani, The limit cycle of the van der Pol equation is not algebraic, J. Differential Equations 115 (1995), 146-152. MR1308609 (95i:34051)

[13] M. F. Singer, Liouvillian first integrals of differential systems, Trans. Amer. Math. Soc. 333 (1992), 673-688. MR:1062869 (92m:12014)

Departament de Matemàtiques, Universitat Autònoma de Barcelona, 08193 Bellaterra, Barcelona, Catalonia, Spain

E-mail address: jllibre@mat.uab.cat

Departamento de Matemática, Instituto Superior Técnico, Av. Rovisco Pais 1049-001, Lisboa, Portugal

E-mail address: cvalls@math.ist.utl.pt 